\title{
Morbilidad y mortalidad perinatal de hijos de mujeres con enfermedad renal crónica
}

\author{
Ma. Juana Pérez-López, ${ }^{1}$ Isabel G. Leyva-Reséndiz, ${ }^{2}$ Benjamín Vázquez-Vega, ${ }^{1}$ \\ Ernesto L. Chávez-López, ${ }^{1}$ Juan C. Hernández-Rivera ${ }^{3 *}$ y José R. Paniagua-Sierra ${ }^{3}$ \\ ${ }^{1}$ Centro Médico Nacional La Raza, Hospital de Especialidades; ${ }^{2}$ Centro Médico Nacional La Raza, Hospital de Ginecología y Obstetricia; ${ }^{3}$ Centro
} Médico Nacional Siglo XXI, Hospital de Especialidades. Instituto Mexicano del Seguro Social, Ciudad de México, México

\begin{abstract}
Resumen
Introducción: La enfermedad renal (ER) crónica asociada al embarazo incrementa el riesgo de complicaciones maternas y fetales. Objetivo: Determinar la morbilidad y mortalidad perinatal del hijo de madre con enfermedad renal leve y moderada del embarazo. Métodos: Estudio retrospectivo de expedientes de mujeres con ER leve y moderada del embarazo atendidas en el Centro Médico Nacional La Raza entre 2010 y 2016. Resultados: Se trató de 142 pacientes, 99 (69.72 \%) con ER leve y 43 (30.28 \%) con ER moderada; 79 (55.63 \%) neonatos llegaron a término, 28 (19.71\%) presentaron restricción de crecimiento; 44 (30.98\%), peso bajo al nacimiento y 54 (38.02\%) ingresaron a la unidad de cuidados intensivos neonatales; cuatro (4.04\%) mujeres presentaron aborto, en cuatro (2.81\%) sus hijos presentaron muerte intrauterina y en 10 (7.04\%), muerte neonatal. La presión arterial alta $(R M=6.93)$ y la hemoglobina $<11 \mathrm{~g} / \mathrm{dL}(R M=2.48)$ constituyeron factores de riesgo para prematurez. Conclusión: Se encontró relación entre la anemia y las cifras de tensión arterial como riesgo para prematurez, Apgar bajo e ingreso a unidad de cuidados intensivos neonatales.
\end{abstract}

PALABRAS CLAVE: Enfermedad renal leve. Enfermedad renal moderada. Embarazo. Mortalidad perinatal.

\section{Perinatal morbidity and mortality of children born to mothers with chronic kidney disease}

\begin{abstract}
Introduction: Chronic kidney disease (CKD) associated with pregnancy increases the risk of maternal and fetal complications. Objective: To determine perinatal morbidity and mortality of children born to mothers with mild and moderate CKD during pregnancy. Methods: Retrospective study of medical records of women with mild and moderate CKD during pregnancy cared for at La Raza National Medical Center between 2010 and 2016. Results: There were 142 patients, 99 (69.72\%) with mild CKD and 43 (30.28 \%) with moderate CKD; 79 neonates (55.63\%) reached full term, 28 (19.71\%) had growth restriction; $44(30.98 \%)$, low birth weight and 54 (38.02\%) were admitted to the neonatal intensive care unit (NICU); four women (4.04\%) had an abortion; in four (2.81\%), their children had intrauterine death, and in 10 (7.04\%), neonatal death. High blood pressure $(O R=6.93)$ and hemoglobin $<11 \mathrm{~g} / \mathrm{dL}(O R=2.48)$ were risk factors for prematurity. Conclusion: $A$ relationship was found between anemia and blood pressure levels and risk for prematurity, low Apgar and NICU admission.
\end{abstract}

KEY WORDS: Mild kidney disease. Moderate kidney disease. Pregnancy. Perinatal mortality. 


\section{Introducción}

La incidencia de enfermedad renal (ER) crónica asociada al embarazo es de aproximadamente 0.03 a $0.12 \%$ según registros en población norteamericana y de $0.33 \%$ conforme un estudio mexicano. ${ }^{1,2}$ Esta asociación incrementa el riesgo de complicaciones maternas y fetales. Las complicaciones fetales más frecuentes son retardo en el crecimiento intrauterino, bajo peso al nacimiento, mayor estancia intrahospitalaria y muerte perinatal. ${ }^{1-3}$

La creatinina sérica aún es el parámetro de referencia para evaluar la función renal en las mujeres embarazadas. La clasificación de Davison-Lindheimer en la gestación divide a las embarazada con ER en tres categorías según los niveles de creatinina sérica:

- Con ER leve, creatinina $<1.4 \mathrm{~mL}$.

- Con ER moderada, creatinina entre 1.4 y $2.8 \mathrm{mg} / \mathrm{dL}$.

- Con ER severa, creatinina $>2.8 \mathrm{mg} / \mathrm{dL} .{ }^{4,5}$

Entre los efectos de la ER sobre el embarazo se encuentra el incremento de los resultados adversos fetales, el cual se correlaciona con la severidad de la ER antes de la gestación. ${ }^{6,7}$ En un estudio de 38 mujeres embarazadas con ER, Bar et al. describieron prematurez en $22 \%$ y restricción del crecimiento intrauterino (RCIU) en $13 \% .^{8,9}$ En 67 pacientes con filtrado glomerular $<60 \mathrm{~mL} /$ minuto/1.73 $\mathrm{m}^{2}$, Jones y Hayslett encontraron prematurez y RCIU en $59 \%$, así como supervivencia fetal en $93 \% \cdot{ }^{10-12}$ En un metaanálisis, Nevis et al. identificaron peores resultados fetales en mujeres con ER respecto a aquellas sin esta enfermedad, como parto prematuro, talla baja para la edad gestacional y bajo peso al nacimiento, con razón de momios (RM) de 1.8, 2.67 y 4.85 , respectivamente..$^{13,14}$ La supervivencia fetal y neonatal es menor cuando las cifras de tensión arterial están descontroladas, con y sin proteinuria (> $500 \mathrm{mg} / \mathrm{día}$ ), como han señalado Lindheimer y Katz. ${ }^{15-23}$

El objetivo del presente estudio fue determinar la morbilidad y mortalidad perinatal de recién nacidos de mujeres con enfermedad renal leve y moderada del embarazo.

\section{Métodos}

Se analizó una cohorte retrospectiva de 142 recién nacidos de mujeres con ER crónica leve y moderada, atendida entre el 1 de enero de 2010 y el 31 de diciembre de 2016 en el Centro Médico Nacional La Raza; con base en la creatinina sérica se estratificaron conforme la clasificación de DavisonLindheimer (Anexo 1).

Los datos demográficos y clínicos se obtuvieron de los expedientes médicos. De las mujeres se capturó la semana de diagnóstico del embarazo, comorbilidades y tiempo de evolución de la ER. De los recién nacidos se registró disminución del crecimiento intrauterino (debajo del percentil 3), bajo peso al nacimiento $<2500 \mathrm{~g}$ (Anexo 2), óbito fetal e ingreso a la unidad de cuidados intensivos neonatales (UCIN).

Los datos se expresan como frecuencias, medianas y rangos intercuartilares o promedios \pm desviaciones estándar, según las características de las variables. Las diferencias entre los grupos se analizaron con $\chi^{2}$ o t de Student, según el caso. Para establecer riesgos se hizo cálculo de regresión logística binaria y múltiple. Se empleó el programa Statistical Package for the Social Sciences versión 25.

\section{Resultados}

Entre enero de 2010 y diciembre de 2016 se registraron 142 recién nacidos de mujeres con ER crónica leve y moderada del embarazo. El grupo de recién nacidos de mujeres con ER leve estuvo integrado por 99 sujetos $(69.72 \%)$ y el de mujeres con ER moderada, por $43(30.28 \%)$.

Las características basales de las mujeres embarazadas que influyeron en los desenlaces fetales están descritas en la Tabla 1. El promedio de edad fue de 29.2 años, con una mínima de 18 y una máxima de 44; la proporción de pacientes que presentaron comorbilidades fue de $57.57 \%$ en el grupo con ER leve y de $55.81 \%$ en el grupo con ER moderada. Las indicaciones maternas y fetales para la resolución del embarazo se muestran en la Tabla 2.

La principal indicación para la interrupción del embarazo fue la preeclampsia con datos de severidad, la segunda fue la ER crónica y la tercera difirió en los dos grupos: en las pacientes con ER leve fue el trabajo de parto en fase activa $(n=8,8.08 \%)$ y en el grupo con ER moderada, el deterioro de la función renal $(n=7,16.27 \%)$. La cesárea fue la forma más utilizada para la interrupcion del embarazo.

La edad gestacional fue calculada mediante el método de Capurro: mediana de 35.31 semanas en el grupo de mujeres con ER leve y 34.39 semanas en el grupo con ER moderada. El peso promedio de los recién nacidos de las mujeres con ER leve fue de $2423 \mathrm{~g}$ y de $2140 \mathrm{~g}$ en los hijos de las mujeres con 
Tabla 1. Características basales de las mujeres durante el embarazo

\begin{tabular}{|c|c|c|c|c|c|}
\hline \multirow[t]{2}{*}{ Características } & \multicolumn{2}{|c|}{ ER leve $(n=99)$} & \multicolumn{2}{|c|}{ ER moderada $(n=43)$} & \multirow[t]{2}{*}{$\mathbf{p}^{*}$} \\
\hline & Mediana & Min., Máx. & Mediana & Min., Máx. & \\
\hline Edad (años) & 29.45 & 18,44 & 28.65 & 18,40 & 0.664 \\
\hline Gestas & 2.07 & 1,5 & 1.93 & 1,4 & 0.513 \\
\hline Partos & 0.33 & 0,2 & 0.37 & 0,2 & 0.682 \\
\hline Abortos & 0.38 & 0,2 & 0.34 & 0,2 & 0.945 \\
\hline Cesáreas & 1.32 & 0,3 & 1.11 & 0,3 & 0.164 \\
\hline Hemoglobina (g/dL) & 11.97 & $9.3,16.8$ & 11.40 & $9,16.5$ & 0.450 \\
\hline Albúmina (mg/dL) & 3.14 & $1.8,6.6$ & 3.01 & $1.4,4.7$ & 0.380 \\
\hline Creatinina (mg/dL) & 0.95 & $0.5,1.5$ & 1.84 & $1.4,2.7$ & $<0.001$ \\
\hline Urea (mg/dL) & 29.75 & $11,107.9$ & 55.95 & $20.8,126$ & $<0.001$ \\
\hline Depuración de $\mathrm{Cr}$ (mL/minuto) & 86.98 & $24,188.7$ & 48.53 & $13.87,188$ & $<0.001$ \\
\hline Depuración de Cr (CKD-EPI) & 83.34 & $45.3,138.3$ & 39.04 & $21.3,54.7$ & $<0.001$ \\
\hline \multirow[t]{2}{*}{ Proteinuria (g) } & 0.571 & $0,6.6$ & 1.97 & $0.025,9.62$ & 0.086 \\
\hline & $n$ & $\%$ & $\mathrm{n}$ & $\%$ & \\
\hline Sin proteinuria $(<300 \mathrm{mg})$ & 79 & 79.79 & 24 & 55.81 & \\
\hline Proteinuria $300 \mathrm{mg}$ a $1 \mathrm{~g}$ & 6 & 6.06 & 8 & 18.60 & \\
\hline Proteinuria $\geq 1 \mathrm{~g}$ & 14 & 14.14 & 11 & 25.58 & \\
\hline $\begin{array}{l}\text { Comorbilidades } \\
\text { No diabetes mellitus } \\
\text { Diabetes gestacional } \\
\text { DM1 } \\
\text { DM2 } \\
\text { No HAS } \\
\text { Hipertensión gestacional } \\
\text { HAS } \\
\text { Lupus eritematoso sistémico }\end{array}$ & $\begin{array}{c}57 \\
92 \\
1 \\
5 \\
1 \\
55 \\
28 \\
16 \\
5\end{array}$ & $\begin{array}{c}57.57 \\
92.92 \\
1.01 \\
5.05 \\
1.01 \\
55.55 \\
28.28 \\
16.16 \\
5.05\end{array}$ & $\begin{array}{c}24 \\
38 \\
0 \\
5 \\
0 \\
22 \\
5 \\
16 \\
3\end{array}$ & $\begin{array}{c}55.81 \\
88.37 \\
11.62 \\
\\
51.16 \\
11.62 \\
37.20 \\
6.97\end{array}$ & \\
\hline ER diagnosticada $<20$ SDG & 23 & 23.23 & 11 & 25.58 & \\
\hline ER diagnosticada > 20 SDG & 47 & 47.47 & 19 & 44.18 & \\
\hline
\end{tabular}

ER moderada. El peso se ajustó por percentil según la edad gestacional dada la relación directa entre ambas características (Anexo 2).

Llegaron a término $55.63 \%$ de los embarazos, $35.91 \%$ de los hijos fueron prematuros (de 28.1 a 36.6 semanas de gestación) y $4.92 \%$ (<28.1 semanas), prematuros extremos. Se presentó restricción del crecimiento intrauterino (RCIU) en $19.71 \%$, bajo peso para la edad gestacional en $30.88 \%$, Apgar indicativo de depresión moderada en $3.52 \%$, Apgar indicativo de depresión severa en $6.33 \%$ e ingreso a la UCIN en $38.02 \%$ (Tabla 3).
La supervivencia global de los productos fue de $86.04 \%$ en el grupo de los hijos de madres con ER moderada y de $86.86 \%$ en los hijos de madres con ER leve, con un total de cuatro abortos (4.04\%) en el grupo de ER leve y uno (2.32\%) en el grupo con ER moderada. Se realizaron regresiones logísticas multivariadas de cada una de las variables independientes respecto al desenlace perinatal: la presencia de anemia (hemoglobina $<11 \mathrm{~g} / \mathrm{dL}$ ) aumentó 2.92 veces el riesgo de un aborto $(\mathrm{RM}=2.92$, intervalo de confianza $[\mathrm{IC}] \mathrm{de}$ $95 \%=0.45-18.3, p=0.22$ ) y la presencia de ER 
Tabla 2. Indicaciones de interrupción del embarazo

\begin{tabular}{|c|c|c|c|c|c|c|}
\hline \multirow[t]{2}{*}{ Indicaciones } & \multicolumn{2}{|c|}{ ER leve } & \multicolumn{2}{|c|}{ ER moderada } & \multicolumn{2}{|c|}{ Total } \\
\hline & $n$ & $\%$ & $\mathbf{n}$ & $\%$ & $n$ & $\%$ \\
\hline $\begin{array}{l}\text { Maternas } \\
\text { Enfermedad renal } \\
\text { Deterioro de la función renal } \\
\text { Preeclampsia con datos de severidad } \\
\text { Trabajo de parto fase activa } \\
\text { Falta de progresión de trabajo de parto } \\
\text { Desproporción cefalopélvica } \\
\text { Periodo intergenésico corto } \\
\text { Embarazo gemelar } \\
\text { Embarazo en presentación pélvica } \\
\text { Cesárea iterativa } \\
\text { Antecedente de cirugía uterina } \\
\text { Placenta previa } \\
\text { Oligohidramnios }\end{array}$ & $\begin{array}{c}19 \\
2 \\
33 \\
8 \\
3 \\
3 \\
1 \\
0 \\
2 \\
4 \\
1 \\
4 \\
2\end{array}$ & $\begin{array}{c}19.19 \\
2.02 \\
33.33 \\
8.08 \\
3.03 \\
3.03 \\
1.01 \\
0 \\
2.02 \\
4.04 \\
1.01 \\
4.04 \\
2.02\end{array}$ & $\begin{array}{l}8 \\
7 \\
15 \\
2 \\
0 \\
4 \\
0 \\
1 \\
1 \\
1 \\
0 \\
0 \\
2\end{array}$ & $\begin{array}{c}18.60 \\
16.27 \\
34.84 \\
4.65 \\
0 \\
9.30 \\
0 \\
2.32 \\
2.32 \\
2.32 \\
0 \\
0 \\
4.65\end{array}$ & $\begin{array}{c}27 \\
9 \\
48 \\
10 \\
3 \\
7 \\
1 \\
1 \\
3 \\
5 \\
1 \\
4 \\
4\end{array}$ & $\begin{array}{c}19.01 \\
6.33 \\
33.80 \\
7.04 \\
2.11 \\
4.92 \\
0.70 \\
0.70 \\
2.11 \\
3.52 \\
0.70 \\
2.81 \\
2.81\end{array}$ \\
\hline $\begin{array}{l}\text { Fetales } \\
\text { Aborto } \\
\text { Óbito } \\
\text { Baja reserva fetal } \\
\text { Restricción del crecimiento intrauterino }\end{array}$ & $\begin{array}{l}3 \\
1 \\
8 \\
5\end{array}$ & $\begin{array}{l}3.03 \\
1.01 \\
8.08 \\
5.05\end{array}$ & $\begin{array}{l}1 \\
1 \\
0 \\
0\end{array}$ & $\begin{array}{c}2.32 \\
2.32 \\
0 \\
0\end{array}$ & $\begin{array}{l}4 \\
2 \\
8 \\
5\end{array}$ & $\begin{array}{l}2.81 \\
1.40 \\
5.63 \\
3.52\end{array}$ \\
\hline
\end{tabular}

Tabla 3. Vía de interrupción del embarazo y resultados perinatales

\begin{tabular}{|c|c|c|c|c|c|c|}
\hline \multirow[t]{2}{*}{ Vía de interrupción } & \multicolumn{2}{|c|}{ ER leve } & \multicolumn{2}{|c|}{ ER moderada } & \multicolumn{2}{|c|}{ Total } \\
\hline & $\mathrm{n}$ & $\%$ & $\mathrm{n}$ & $\%$ & $\mathrm{n}$ & $\%$ \\
\hline $\begin{array}{l}\text { Vía abdominal cesárea } \\
\text { Parto } \\
\text { Legrado } \\
\text { Total }\end{array}$ & $\begin{array}{c}82 \\
13 \\
4 \\
99\end{array}$ & $\begin{array}{c}82.82 \\
13.13 \\
4.04 \\
100\end{array}$ & $\begin{array}{c}37 \\
6 \\
0 \\
43\end{array}$ & $\begin{array}{c}86.04 \\
13.95 \\
0 \\
100\end{array}$ & $\begin{array}{c}119 \\
19 \\
4 \\
142\end{array}$ & $\begin{array}{c}83.80 \\
13.38 \\
2.81 \\
100\end{array}$ \\
\hline Resultados perinatales & Mediana & Máx--Mín. & Mediana & Máx.-Mín. & \multicolumn{2}{|c|}{$\mathbf{p}$} \\
\hline Peso & 2423.59 & $250-4000$ & 2140.81 & $300-3350$ & \multicolumn{2}{|c|}{$0.177^{\star}$} \\
\hline Semanas de gestación & 35.31 & $10-42$ & 34.39 & $21-40$ & \multicolumn{2}{|c|}{$0.023^{*}$} \\
\hline Apgar a los 5 minutos & 8.6 & $5-10$ & 8.6 & $5-9$ & & \\
\hline Días de ElH & 9.12 & $0-131$ & 14.88 & $0-83$ & \multicolumn{2}{|c|}{$0.681^{*}$} \\
\hline Días de EIH en UCIN & 3.5 & $0-131$ & 2 & $0-27$ & \multicolumn{2}{|c|}{$0.91^{*}$} \\
\hline
\end{tabular}

$\mathrm{ER}=$ enfermedad renal, $\mathrm{EIH}=$ estancia intrahospitalaria, $\mathrm{UCIN}=$ unidad de cuidados intensivos neonatales.

*U de Mann-Whitney.

aumentó 1.76 veces el riesgo de un aborto (IC $95 \%=0.19-16.3, p=0.52)$ (Tabla 4).

Respecto a las variables independientes con significación estadística, se observó que la tensión arterial descontrolada aumentó 93 veces el riesgo de obtener un producto prematuro (IC $95 \%=1.43-33.4$, $p=0.0092)$ respecto a finalizar a término el embarazo y que la anemia (hemoglobina $<11 \mathrm{~g} / \mathrm{dL}$ ) lo aumentó 6. 2.48 veces (IC $95 \%=1.21-5.11$, $p=0.017$ ); la EC leve y la moderada mostraron tendencia de riesgo de prematurez de 1.88 veces (IC $95 \%=0.90-3.90, p=0.08$ ), sin tener una significación estadística y la proteinuria, 1.4 veces (IC $95 \%=0.66-2.95, p=0.37$ ). 
Tabla 4. Análisis multivariado de las variables de estudio con el desenlace del embarazo en pacientes con ER crónica

\begin{tabular}{|c|c|c|c|c|c|c|c|c|c|c|}
\hline Variables & $\begin{array}{c}\text { Abortos } \\
n(\%)\end{array}$ & $\begin{array}{c}\text { Nacimientos } \\
n(\%)\end{array}$ & RM & IC $95 \%$ & $\mathrm{p}^{*}$ & $\begin{array}{c}\text { Prematuro } \\
n(\%)\end{array}$ & $\begin{array}{c}\text { A término } \\
n(\%)\end{array}$ & $\mathbf{R M}$ & IC $95 \%$ & $\mathbf{p}^{*}$ \\
\hline $\begin{array}{l}\text { Enfermedad renal } \\
\text { crónica } \\
\text { Leve } \\
\text { Moderada }\end{array}$ & $\begin{array}{l}4(4.04) \\
1(2.33)\end{array}$ & $\begin{array}{l}95(95.96) \\
42(97.67)\end{array}$ & 1.76 & $0.19-16.3$ & 0.52 & $\begin{array}{l}23(53.5) \\
36(37.9)\end{array}$ & $\begin{array}{l}20(46.5) \\
59(62.1)\end{array}$ & 1.88 & $0.90-3.9$ & $0.0864^{* *}$ \\
\hline $\begin{array}{l}\text { Proteinuria } \\
\text { Con proteinuria } \\
\text { Sin proteinuria }\end{array}$ & $\begin{array}{c}0(0) \\
5(4.85)\end{array}$ & $\begin{array}{c}39(100) \\
98(95.15)\end{array}$ & - & - & - & $\begin{array}{l}19(48.7) \\
40(40.4)\end{array}$ & $\begin{array}{l}20(51.3) \\
59(59.6)\end{array}$ & 1.4 & $0.66-2.95$ & $0.374^{* *}$ \\
\hline $\begin{array}{l}\text { Cifras de tensión arterial } \\
\text { Normal } \\
\text { Elevada }\end{array}$ & $\begin{array}{l}4(3.08) \\
1(8.33)\end{array}$ & $\begin{array}{c}126(96.92) \\
11(91.67)\end{array}$ & 0.34 & $0.04-3.4$ & 0.361 & $\begin{array}{c}9(81.8) \\
50(39.4)\end{array}$ & $\begin{array}{c}2(18.2) \\
77(60.6)\end{array}$ & 6.93 & $1.43-33.4$ & $0.0092^{* *}$ \\
\hline $\begin{array}{l}\text { Anemia } \\
\text { Anemia } \\
\text { Sin anemia }\end{array}$ & $\begin{array}{l}3(6.12) \\
2(2.15)\end{array}$ & $\begin{array}{l}46(93.88) \\
91(97.85)\end{array}$ & 2.92 & $0.45-18.38$ & 0.22 & $\begin{array}{l}27(57.4) \\
32(35.2)\end{array}$ & $\begin{array}{l}20(42.6) \\
59(64.8)\end{array}$ & 2.48 & $1.21-5.11$ & $0.017^{* *}$ \\
\hline & $\begin{array}{l}\text { Depresión } \\
\text { mod. y sev. } \\
\text { n (\%) }\end{array}$ & $\begin{array}{l}\text { Sin } \\
\text { depresión } \\
n(\%)\end{array}$ & RM & IC $95 \%$ & $\mathbf{p}^{*}$ & $\begin{array}{l}\text { RCIU } \\
\text { n (\%) }\end{array}$ & $\begin{array}{c}\text { Sin RCIU } \\
\mathbf{n}(\%)\end{array}$ & RM & IC $95 \%$ & $\mathbf{p}^{*}$ \\
\hline $\begin{array}{l}\text { Enfermedad renal } \\
\text { crónica } \\
\text { Leve } \\
\text { Moderada }\end{array}$ & $\begin{array}{c}5(11.6) \\
4(4.2)\end{array}$ & $\begin{array}{l}38(88.4) \\
91(95.8)\end{array}$ & 2.99 & $0.76-11.75$ & 0.13 & $\begin{array}{c}5(11.6) \\
23(23.2)\end{array}$ & $\begin{array}{l}38(88.4) \\
76(76.8)\end{array}$ & 0.43 & $0.15-1.23$ & 0.11 \\
\hline $\begin{array}{l}\text { Proteinuria } \\
\text { Con proteinuria } \\
\text { Sin proteinuria }\end{array}$ & $\begin{array}{l}2(5.1) \\
7(7.1)\end{array}$ & $\begin{array}{l}37(94.9) \\
92(92.9)\end{array}$ & 0.71 & $0.14-3.57$ & 1 & $\begin{array}{l}10(25.6) \\
18(17.5)\end{array}$ & $\begin{array}{l}29(74.4) \\
85(82.5)\end{array}$ & 1.62 & $0.67-3.92$ & $0.27^{\star \star}$ \\
\hline $\begin{array}{l}\text { Cifras de tensión arterial } \\
\text { Normal } \\
\text { Elevada }\end{array}$ & $\begin{array}{c}0(0) \\
9(7.1)\end{array}$ & $\begin{array}{c}11(100) \\
118(92.9)\end{array}$ & - & - & - & $\begin{array}{c}5(41.7) \\
23(17.7)\end{array}$ & $\begin{array}{c}7(58.3) \\
107(82.3)\end{array}$ & 3.32 & $0.96-11.40$ & 0.06 \\
\hline $\begin{array}{l}\text { Anemia } \\
\text { Anemia } \\
\text { Sin anemia }\end{array}$ & $\begin{array}{l}7(14.9) \\
2(2.2)\end{array}$ & $\begin{array}{l}40(85.1) \\
89(97.8)\end{array}$ & 7.78 & $1.54-39.16$ & 0.008 & $\begin{array}{c}9(18.4) \\
19(20.4)\end{array}$ & $\begin{array}{l}40(81.6) \\
74(79.6)\end{array}$ & 0.87 & $0.36-2.11$ & 0.76 \\
\hline & $\begin{array}{l}\text { Bajo peso al } \\
\text { nacimiento } \\
n(\%)\end{array}$ & $\begin{array}{c}\text { Peso } \\
\text { adecuado } \\
\text { n (\%) }\end{array}$ & RM & IC $95 \%$ & $p^{*}$ & $\begin{array}{l}\text { Ingreso a } \\
\text { UCIN } \\
n(\%)\end{array}$ & $\begin{array}{l}\text { No } \\
\text { ingreso a } \\
\text { UCIN } \\
n(\%)\end{array}$ & RM & IC $95 \%$ & $p^{*}$ \\
\hline $\begin{array}{l}\text { Enfermedad renal } \\
\text { crónica } \\
\text { Leve } \\
\text { Moderada }\end{array}$ & $\begin{array}{l}12(27.9) \\
32(32.3)\end{array}$ & $\begin{array}{l}31(72.1) \\
67(67.7)\end{array}$ & 1.234 & $0.56-2.71$ & $0.6^{\star *}$ & $\begin{array}{c}6(14) \\
17(17.2)\end{array}$ & $\begin{array}{c}37(86) \\
82(82.8)\end{array}$ & 0.78 & $0.28-2.14$ & 0.63 \\
\hline $\begin{array}{l}\text { Proteinuria } \\
\text { Con proteinuria } \\
\text { Sin proteinuria }\end{array}$ & $\begin{array}{l}15(38.5) \\
29(28.2)\end{array}$ & $\begin{array}{l}24(61.5) \\
74(71.8)\end{array}$ & 1.595 & $0.73-3.46$ & $0.23^{\star \star}$ & $\begin{array}{c}7(17.9) \\
16(15.5)\end{array}$ & $\begin{array}{l}32(82.1) \\
87(84.5)\end{array}$ & 1.18 & $0.44-3.15$ & 0.72 \\
\hline $\begin{array}{l}\text { Cifras de tensión arterial } \\
\text { Normal } \\
\text { Elevada }\end{array}$ & $\begin{array}{l}5(41.7) \\
39(30)\end{array}$ & $\begin{array}{l}7(58.3) \\
91(70)\end{array}$ & 1.66 & $0.49-5.57$ & 0.4 & $\begin{array}{c}5(41.7) \\
18(13.8)\end{array}$ & $\begin{array}{c}7(58.3) \\
112(86.2)\end{array}$ & 4.44 & $1.27-15.52$ & 0.012 \\
\hline $\begin{array}{l}\text { Anemia } \\
\text { Anemia } \\
\text { Sin anemia }\end{array}$ & $\begin{array}{l}16(32.7) \\
28(30.1)\end{array}$ & $\begin{array}{l}33(67.3) \\
65(69.9)\end{array}$ & 1.12 & $0.53-2.36$ & $0.75^{\star *}$ & $\begin{array}{c}9(18.4) \\
14(15.1)\end{array}$ & $\begin{array}{l}40(81.6) \\
79(84.9)\end{array}$ & 1.26 & $0.50-3.1$ & 0.61 \\
\hline
\end{tabular}

*Prueba exacta de Fisher, ${ }^{\star *}$ Prueba de $\chi^{2}, \mathrm{RM}=$ razón de momios, IC = intervalo de confianza, UCIN = unidad de cuidados intensivos neonatales, RCIU = restricción del crecimiento intrauterino.

La anemia fue estadísticamente significativa $(\mathrm{RM}=7.78, \mathrm{IC}=1.54-39.16, \mathrm{p}=0.008)$ en mostrar una disminución de la puntuación de Apgar a los cinco minutos y la ER estuvo relacionada 2.99 veces con el riesgo de Apgar indicativo de depresión moderada a severa (IC $95 \%=0.76-11.75, p=0.130)$. 
El descontrol de la tensión arterial aumentó 3.32 veces el riesgo de RCIU (IC $95 \%=0.96-11.40$, $\mathrm{p}=0.060$ ). La elevación de las cifras de la presión arterial aumentó 4.44 veces el riesgo de ingreso a la UCIN.

\section{Discusión}

De las pacientes con diagnóstico de ER y embarazo, 99 (69.72\%) se encontraron en el grupo de ER leve y $43(30.28 \%)$ en el grupo de ER moderada, similar a lo reportado en la literatura, en la cual se refiere que la mayor proporción de pacientes presenta ER leve del embarazo.

El peso registrado al nacimiento de los hijos de madres con ER leve fue de $2423 \mathrm{~g}$ (250-4000 g) y el de los hijos de madres con ER moderada fue de $2140 \mathrm{~g}$ (300-3350 g), con una diferencia de $283 \mathrm{~g}$ entre las medianas de ambos grupos, no estadísticamente significativa con una $p=0.177$ mediante la prueba de $U$ de Mann-Whitney. En las mujeres con ER leve se registró $63.63 \%$ de recién nacidos con peso adecuado para la edad gestacional, $32.32 \%$ con peso bajo para la edad gestacional y $1.01 \%$ con peso alto para la edad gestacional. En las mujeres con ER moderada se registró $72.09 \%$ de recién nacidos con peso adecuado para la edad gestacional y $27.90 \%$ con peso bajo.

En ambos grupos se registró $19.71 \%$ de RCIU, en el grupo con ER leve fue de $23.23 \%$ y en el grupo con ER moderada de $11.62 \%$, proporción ligeramente mayor a la reportada por Trevisan et al., quienes indicaron $13 \%$, y menor a la informada por Jones y Hayslett, quienes refirieron $37 \%$ de RCIU, y por Cunningham, quien señaló $35 \%$.

La edad gestacional al nacimiento fue estadísticamente significativa entre ambos grupos: en las mujeres con ER leve, la mediana fue de 35.31 semanas y en las mujeres con ER moderada fue de 34.39 semanas, con una $p=0.023$. En las mujeres con ER leve se identificó $59.59 \%$ de embarazos a término, $32.32 \%$ de prematuros entre las 28.1 y 36.6 semanas de gestación y $4.04 \%$ de prematuros extremos. En el grupo con ER moderada hubo $46.51 \%$ de embarazos a término, $44.18 \%$ de prematuros entre las 28.1 y 36.6 semanas de gestación y $9.30 \%$ de prematuros extremos. En ambos grupos se encontró $55.63 \%$ de embarazos a término y $44.37 \%$ de nacidos prematuros. En general, los recién nacidos prematuros fueron menores a los reportados por Trevisan et al., quienes señalaron $60 \%$ de prematuros, mientras que Jones y Hayslett informaron $59 \%$. Sin embargo, fueron más que los reportados por Cunningham (30\%).

Los días de estancia intrahospitalaria (EIH) y los días de estancia en la UCIN no fueron estadísticamente significativos entre ambos grupos, con una $p=0.681$ para los días de ElH y $p=0.910$ para los días de estancia en la UCIN. En el grupo con ER moderada, $86.04 \%$ de los recién nacidos ingresó a la UCIN, mientras que en el grupo con ER leve solo ingresó $17.17 \%$.

El Apgar a los 5 minutos fue de 7 a 10 (sin depresión) en $90.90 \%$ de los hijos de madres con ER leve y en $88.37 \%$ de los hijos de madres con ER moderada; $4.04 \%$ de los hijos de madres con ER leve mostró depresión moderada (Apgar de 4 a 6) y $2.32 \%$ de los hijos de madres con ER moderada. Se presentó depresión severa (Apgar $\leq 3$ ) en $5.05 \%$ de los hijos de madres con ER leve y en $9.30 \%$ de los hijos de madres con ER moderada.

La supervivencia global de los hijos fue de $86.61 \%$ para ambos grupos, de $86.04 \%$ en el grupo de las madres con ER moderada y de $86.86 \%$ en las madres con ER leve, ligeramente menor a $93 \%$ reportado por Jones y Hayslett y ligeramente mayor a $82 \%$ informado por Jungers et al. después de excluir los abortos de primer trimestre (los cuales fueron tomados en cuenta en nuestro estudio). En el grupo de mujeres con ER leve se registró aborto en $4.04 \%$ y respecto a sus hijos, muerte intrauterina en $1.01 \%$, muerte neonatal precoz en $6.06 \%$ y muerte neonatal tardía en $2.02 \%$; en el grupo de mujeres con ER moderada, aborto en $2.32 \%$, muerte intrauterina en $6.97 \%$, muerte neonatal precoz en $2.32 \%$ y muerte neonatal tardía en $2.32 \%$.

El descontrol de cifras de presión arterial $(p=0.0092)$ y la hemoglobina $<11 \mathrm{~g} / \mathrm{dL}(p=0.017)$ mostraron relación estadísticamente significativa con el nacimiento prematuro. El Apgar $<3$, que indica depresión moderada y severa, fue estadísticamente significativo con los niveles de hemoglobina $<11 \mathrm{~g} / \mathrm{dL}$ $(p=0.008)$.

El ingreso a la UCIN tuvo relación estadísticamente significativa con el descontrol de las cifras de la tensión arterial $(p=0.012)$. El bajo peso al nacer, la RCIU, el aborto, la muerte intrauterina y la muerte neonatal no presentaron relación estadísticamente significativa con el grado de ER (leve, moderada), proteinuria, niveles de hemoglobina y cifras de presión arterial. A diferencia de lo reportado por Zhang et al., quienes refieren que la hipertensión descontrolada y la proteinuria empeoran los resultados fetales, 
en este estudio solo se encontró relación estadísticamente significativa entre el descontrol de las cifras de tensión arterial en relación con el nacimiento de niños prematuros e ingreso a la UCIN. La proteinuria no tuvo relación estadísticamente significativa con los resultados perinatales de morbilidad y mortalidad; $\sin$ embargo, es prudente tomar estos resultados con cautela, ya que difieren con lo reportado en la literatura y podrían estar ocasionados por inadecuada recolección de la orina de 24 horas.

La hemoglobina $<11 \mathrm{~g} / \mathrm{dL}$ tuvo relación estadísticamente significativa con Apgar $<3$ y con el nacimiento de niños prematuros. Lo anterior no ha sido reportado en la literatura, ya que en la mayoría de los estudios se evalúa la hemoglobina materna en pacientes con ER severa del embarazo y no se reporta en pacientes con ER leve y moderada.

\section{Conclusiones}

La edad promedio en ambos grupos fue de 29 años; la causa de la ER se desconoció en $48.48 \%$ de las mujeres con enfermedad leve y en $76.74 \%$ de las mujeres con ER moderada; la principal indicación para la interrupción del embarazo fue la preeclampsia con datos de severidad y la segunda, la ER crónica. La edad gestacional promedio al nacimiento fue de 35.31 y 34.39 semanas y el peso fue de 2423 y $2140 \mathrm{~g}$ en los hijos de las mujeres con ER leve y moderada, respectivamente. A diferencia de los reportado en la literatura, no se encontró relación estadísticamente significativa entre proteinuria y resultados perinatales adversos; sin embargo, la anemia y las cifras de presión arterial elevadas fueron estadísticamente significativas para prematurez, Apgar bajo e ingreso a la UCIN.

Por lo anterior, se deben realizar estudios prospectivos y efectuar de manera protocolizada y multidisciplinaria el seguimiento de la madre y del hijo antes, durante y posterior al embarazo, a fin de mejorar los resultados perinatales.

\section{Conflicto de intereses}

Los autores declaran no tener conflicto de intereses alguno.

\section{Financiamiento}

Los autores no recibieron patrocinio para llevar a cabo este artículo.

\section{Responsabilidades éticas}

Protección de personas y animales. Los autores declaran que para esta investigación no realizaron experimentos en seres humanos ni en animales.

Confidencialidad de los datos. Los autores declaran que han seguido los protocolos de su centro de trabajo sobre la publicación de datos de pacientes.

Derecho a la privacidad y consentimiento informado. Los autores declaran que en este artículo no aparecen datos de pacientes.

\section{Bibliografía}

1. Fischer MJ, Lehnerz SD, Hebert JR, Parikh CR. Kidney disease is an independent risk factor for adverse fetal and maternal. Am J Kidney Dis. 2004; $43: 415-423$

2. Manterola D, Hernández JA, Estrada A. Enfermedad renal crónica durante el embarazo: curso clínico y resultados perinatales en el Instituto Nacional de Perinatología Isidro Espinosa de los Reyes. Perinatol Reprod Hum. 2012;26:147-153.

3. Podymow T, August P, Akbari A. Management of renal disease in pregnancy. Obstet Gynecol Clin North Am. 2010:37:195-210.

4. Davison JM, Lindheimer MD. Pregnancy and chronic kidney disease. Semin Nephrol. 2011;31:86-99

5. Davison JM, Lindheimer MD. Renal disorders. En: Creasy RK, Resnik R, lams JD, editores. Maternal-fetal medicine principles and practice. EE. UU.: Saunders; 2004

6. Hayslett JP. Interaction of renal disease and pregnancy. Kidney Int. 1984;25:579-587.

7. Hernández-Rivera JC, Pérez-López MJ, Corzo-Bermúdez $\mathrm{CH}$, García-Covarrubias L, Bermúdez-Aceves L, Chucuan-Castillo CA, et al. Delayed initiation of hemodialysis in pregnant women with chronic kidney disease: logistical problems impact clinical outcomes. An experience from an emerging country. J Clin Med. 2019;8:475

8. Ramin SM, Vidaeff AC, Yeomans ER, Gilstrap LC. Chronic renal disease in pregnancy. Obstet Gynecol. 2006;108:1531-1538.

9. Trevisan G, Lopes-Ramos JG, Martins-Costa S, Barros EJ. Pregnancy in patients with chronic renal insufficiency at Hospital de Clínicas of Porto Alegre, Brazil. Ren Fail. 2004;26:29-34.

10. Jones DC, Hayslett JP. Outcome of pregnancy in women with moderate or severe renal insufficiency. N Engl J Med. 1996;335:226-332.

11. Holley JL, Bernardini J, Quadri KH, Greenberg A, Laifer SA. Pregnancy outcomes in a prospective matched control study of pregnancy and renal disease. Clin Nephrol. 1996;45:77-82.

12. Jungers $P$, Chauveau D. Pregnancy in renal disease. Kidney Int. 1997:52:871-885

13. Nevis IF, Reitsma A, Dominic A, McDonald S, Thabane L, Akl EA, et al. Pregnancy outcomes in women with chronic kidney disease: a systematic review. Clin J Am Soc Nephrol. 2011;6:2587-2598.

14. Cunningham FG, Cox SM, Harstad TW, Mason Ra, Pritchard JA. Chronic renal disease and pregnancy outcome. Am J Obstet Gynecol. 1990:163:453-459.

15. Zhang JJ, Ma XX, Hao L, Liu LJ, Lv JC, Zhang H. A systematic review and meta-analysis of outcomes of pregnancy in CKD and CKD outcomes in pregnancy. Clin J Am Soc Nephrol. 2015;10:1964-1978.

16. Luders C, Castro MC, Titan SM, de Castro I, Elias RM, Abensur H, et al. Obstetric outcome in pregnant women on long-term dialysis: a case series. Am J Kidney Dis. 2010;56:77-85.

17. Hladunewich MA, Melamad N, Bramham K. Pregnancy across the spectrum of chronic kidney disease. Kidney Int. 2016:89:995-1007.

18. Lindheimer MD, Katz Al. Gestation in women with kidney disease: prognosis and management. Baillieres Clin Obstet Gynaecol. 1994;8:387-404.

19. Munkhaugen J, Lyndersen S, Romundstad PR, Wideroe TE, Vikse BE, Hallan S. Kidney function and future risk for adverse pregnancy outcomes: a population-based study from HUNT II, Norway. Nephrol Dial Transplant. 2009;24:3744-3750.

20. Hou SH, Grossman SD, Madias NE. Pregnancy in women with renal disease and moderate renal insufficiency. Am J Med. 1985;78:185-194.

21. Katz Al, Davidson JM, Hayslett JP, Singson E, Lindheimer MD. Pregnancy in women with kidney disease. Kidney Int. 1980;18:192-206.

22. Epidis K. Pregnancy in women with renal disease. Yes or no? Hippokratia. 2011:15:8-12

23. Guzmán-Solís M, Sánchez-Rodríguez O, Montaño-Martínez A, Peredo-Villa D, Paniagua-Sierra R, Trejo-Villeda M, et al. Desenlaces obstétricos en mujeres embarazadas con enfermedad renal crónica y factores asociados. Ginecol Obstet Mex. 2020;88:230-243. 


\section{Anexos}

Anexo 1. Clasificación de enfermedad renal en el embarazo.

\begin{tabular}{|l|l|}
\hline \multicolumn{2}{|l|}{ Clasificación de Davison-Lindheimer } \\
\hline Enfermedad renal leve & Creatinina sérica $<1.4 \mathrm{mg} / \mathrm{dL}$ \\
\hline Enfermedad renal moderada & Creatinina sérica $>1.4 \mathrm{mg} / \mathrm{dL}-<2.8 \mathrm{mg} / \mathrm{dL}$ \\
\hline Enfermedad renal severa & Creatinina sérica $>2.8 \mathrm{mg} / \mathrm{dL}$ \\
\hline
\end{tabular}

Anexo 2. Gráfico de Jurado-García. Correlación del peso al nacimiento con la edad gestacional.

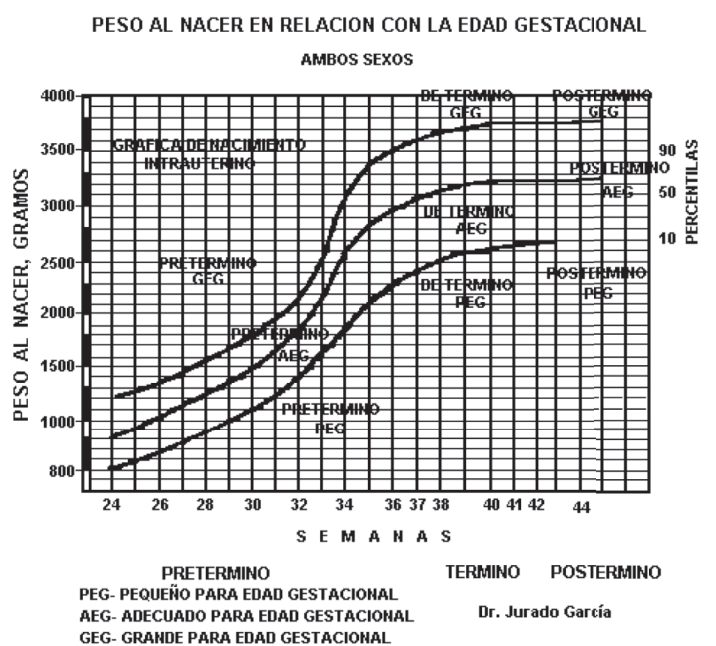

